\title{
Late Twiddler's Syndrome in a Nigerian on a demand pacemaker
}

\author{
Adamu Gati Umar ${ }^{1, ~ *, ~ I b o k ~ I d o n g e s i t ~ O k o n ~}{ }^{1}$, Abdullahi Aishatu², George Alaba Okuku \\ ${ }^{1}$ Department of Medicine, Federal Medical Centre, PMB 14, Efu-Etsuyisa, Bida, Nigeria \\ ${ }^{2}$ Department of Nursing Services, General Hospital, Minna, Nigeria \\ ${ }^{3}$ Department of Haematology and Blood transfusion, Federal Medical Centre, Bida, Nigeria
}

\section{Email address:}

ugadamu@yahoo.com (U. G. Adamu), okon-idyibok@yahoo.com (I. O. Ibok), abdullahiaishatu19@yahoo.com (A. Abdullahi), okukugeorge@ymail.com (A. G. Okuku)

\section{To cite this article:}

Adamu Gati Umar, Ibok Idongesit Okon, Abdullahi Aishatu, George Alaba Okuku. Late Twiddler's Syndrome in a Nigerian on a Demand Pacemaker. Clinical Medicine Research. Vol. 3, No. 5, 2014, pp. 150-152. doi: 10.11648/j.cmr.20140305.18

\begin{abstract}
A 75 year old woman with unipolar ventricular pacemaker presented with cough, difficulty breathing and dizziness. An initial electrocardiogram (ECG) showed atrial fibrillation, incomplete right bundle branch block with no pacemaker spikes. The chest radiograph revealed twisting of the leads at various points. She was managed conservatively with anti-failure drugs and monthly ECG. The pacemaker could not be interrogated due to logistic reasons. However, the ECG during the 8th month of follow-up showed pacemaker spikes and complete left bundle branch block pattern suggestive of spontaneous return of pacemaker function.
\end{abstract}

Keywords: Pacemaker Complication, Arrhythmias, Spontaneous Recovery

\section{Introduction}

Twiddler's syndrome is a clinically significant dislodgement of pacemakers electrode(s) due to rotation of the pulse generator in it pocket. This may cause loss of capture, reduced sensing and occasionally result in lethal dysrrhythmias.

This report is that of a patient with unipolar pacemaker late Twiddler's syndrome with loss of capture and undersensing who was managed conservatively for logistic reasons and whose pacemaker function returned spontaneously without intervention.

\section{Case Presentation}

A 75year old woman who was admitted to the emergency ward with cough, difficulty with breathing and dizziness. No history of fiddling with the pulse generator by the patient or any one close to her. She had a unipolar ventricular pacemaker implanted due to complete heart block 5 years ago in a hospital during the annual pilgrimage where she was said to have suddenly collapsed.

An ECG at presentation showed a heart rate of $66 \mathrm{bpm}$, atrial fibrillation, incomplete right bundle branch block and no pacemaker spikes were seen (fig. 1). 


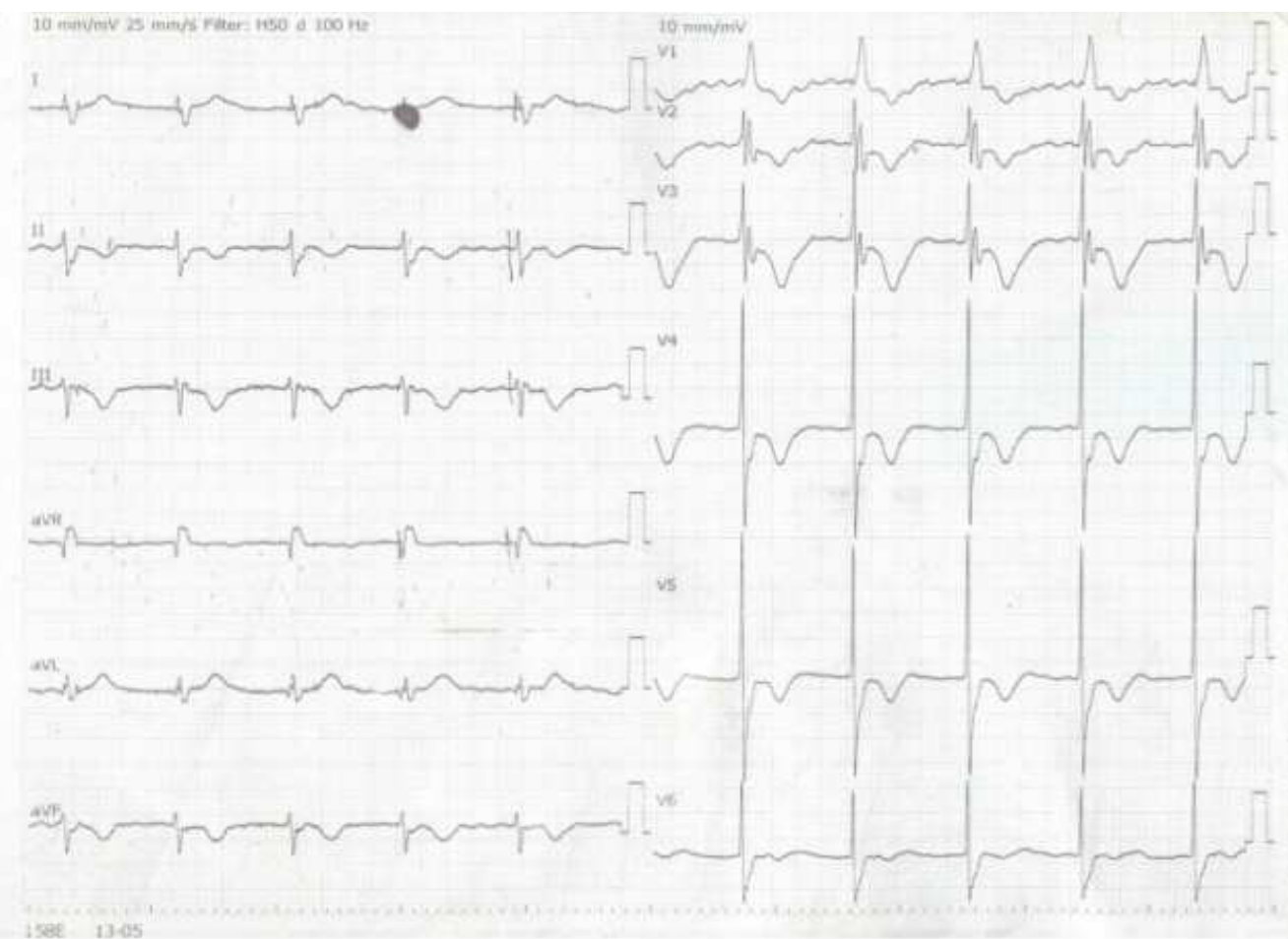

Fig 1. ECG at presentation with no pacemaker spikes

The CXR-AP and Lat. revealed the pulse generator turned upside down and twisting of the electrode at various site (fig 2).

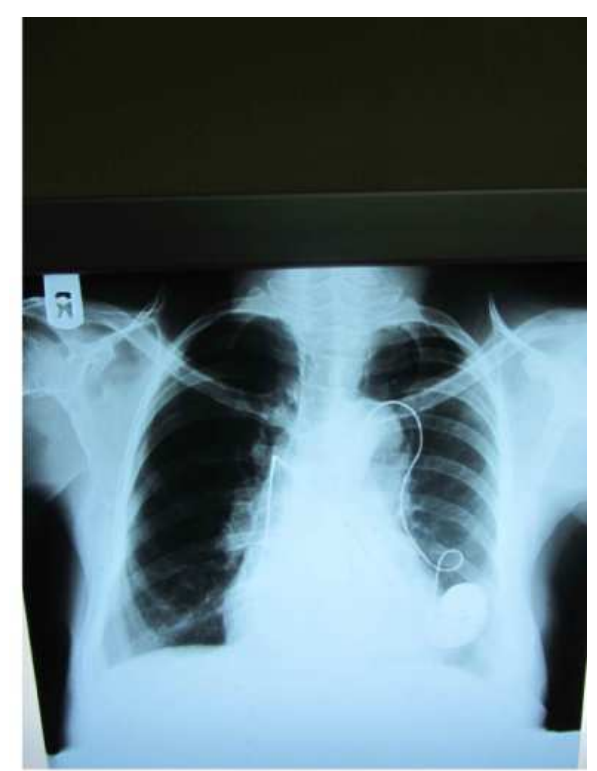

Fig 2. Chestx-pay.PA View showing the twisting of the electrode and generator turned upside down.
She was managed conservatively with anti-failure drugs and was to go for pacemaker interrogation but, could not due to logistic reasons. The monthly ECG at 8 th month of follow-up revealed a heart rate of $60 \mathrm{bpm}$, complete left bundle branch block and ventricular pacemaker spikes (fig. 3) suggestive of spontaneous return of pacemaker function.

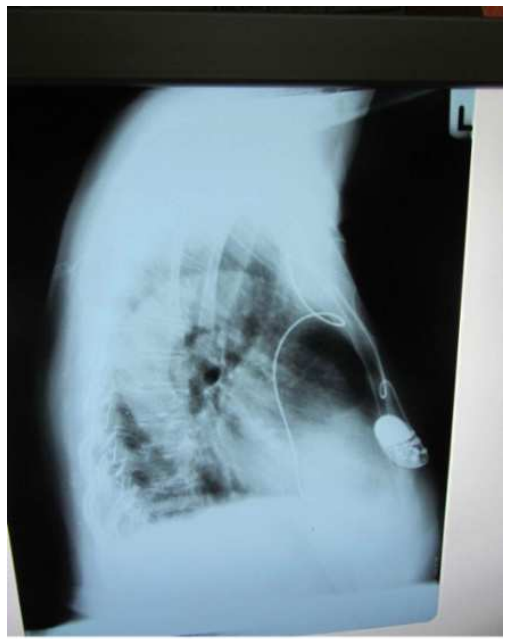

Fig 3. Chest X-Ray lateral View 


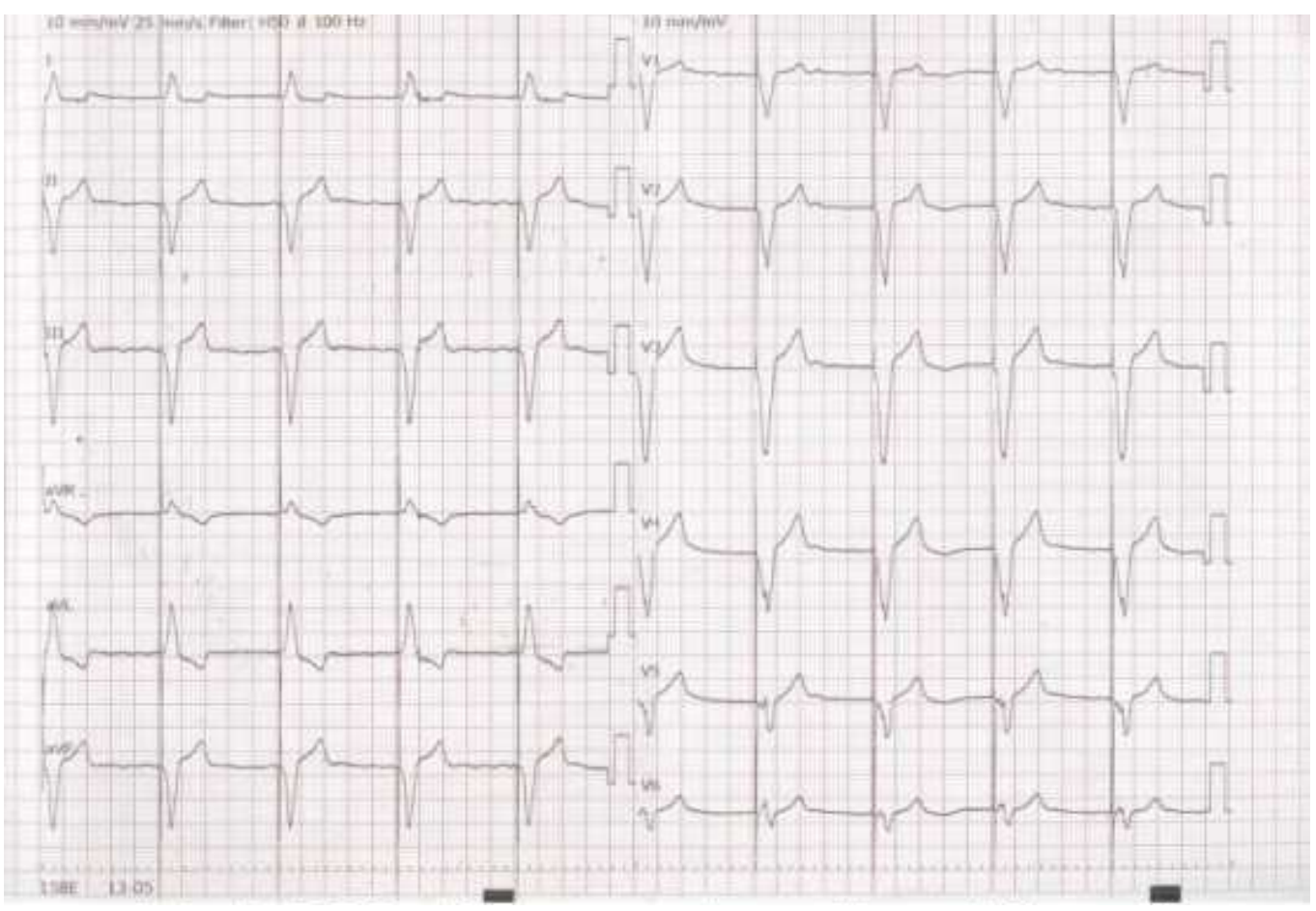

Fig 4. ECG with pacemaker spikes at 8 month of follow-up

\section{Discussion}

Twiddler's syndrome was first described by Bayliss et al both in patients with pacemakers and implantable defibrillators [1]. This describes a permanent malfunction of a pacemaker due to conscious or inadvertent manipulation of the pulse generator on its long axis. It occurs commonly in middle-aged women with too spacious pacemaker pocket and in elderly patients with loose subcutaneous tissues [2-5].

The displacement of the leads may result in under sensing and loss of capture and may stimulate the diaphragm, vagus nerve and pectoralis major and may also wrap round the pulse generator. In extreme cases it may cause dysrrhythmias [6,7].

The patient presented had atrial fibrillation and loss of capture that led to worsening of her symptoms.

Some measures to prevent future occurrence include creating small pocket as well as the use of Dacron patch that promote tissue in-growth during implantation. Others include patient education before discharge and careful follow-up especially in the first few months of implantation $[3,4,7]$.

\section{Conclusion}

Twiddler's syndrome is one of the dreadful complications of pacemaker implantation that can result in loss of function and worsening of symptoms. Restoration of function could be either spontaneous if managed conservatively as in the index case or by re-implantation. Appropriate measures should be put in place to prevent reoccurrence.

\section{References}

[1] Bayliss CE, Beanlands DS, Baird RJ. The pacemakerTwiddler's syndrome: a new complication of implantable transvenous pacemakers. Can Med Assoc J 1968; 99: 371373 .

[2] Nicholson WJ, Tuohy KA, Tilkemeier P. Twiddler's Syndrome. N Engl J Med 2003; 348: 1726-7.

[3] Khalilullah M, Khanna SK, et al. Pacemaker Twiddler's syndrome: a note on its mechanism. $J$ Cardiovasc Surg 1979; 20: $95-100$.

[4] Avitall B, Stormo A, Barragry T, Axtel K, Hare J. Sudden cardiac death: Twiddler's syndrome with an implantable cardioverter defibrillator. Am Heart J 1994; 128: 833-836.

[5] Beatriz Fuertes, Jorge Toquero, Ramón Arroyo-Espliguero, Ignacio F. Lozano. Pacemaker Lead Displacement: Mechanisms and Management. Ind Pacing and Electrophysiology J 2003 3(4): 231-238.

[6] Kumar A, Mckay CR, Rahimtoola SH. Pacemaker Twiddler's Syndrome: an important cause of diaphragmatic pacing. Am J Cardiol 1985; 56: 797-799.

[7] M. Zamirian, M.B. Sharif Kazemi,A.R. Moarref. Pacemaker Twiddler's Syndrome. Iran J Med Sci 2006; 31(1): 59-60. 